\title{
Desarrollo de objetos de aprendizaje para el aprendizaje de las estructuras de datos
}

\author{
Development of learning objects for the learning of data structures
}

Irene Aguilar Juárez

Universidad Autónoma del Estado de México (UAEM), México

ireneico@gmail.com

Víctor Alfonso Alejo Saldívar

Universidad Autónoma del Estado de México (UAEM), México

victor_alejo09@outlook.com

Joel Ayala de la Vega

Universidad Autónoma del Estado de México (UAEM), México

jayalad@uaemex.mx

\section{RESUMEN}

En este trabajo se describe el desarrollo y evaluación de varios objetos de aprendizaje que se usarán como componentes de un curso MOOC (massive open online course) en el marco de trabajo de las comunidades digitales de producción gestionadas por la red CODAES (Comunidades Digitales para el Aprendizaje en Educación Superior) en México. Los materiales surgen ante la necesidad de proveer a los alumnos de nivel superior de profesiones afines a la computación diferentes medios de aprendizaje sobre temas complejos y necesarios en su formación. Los índices de reprobación actuales muestran que las técnicas de enseñanza tradicionales no han resultado efectivas para lograr el aprendizaje de algunos tópicos como la programación; los datos confirman que esta competencia es difícil de adquirir para los estudiantes, por lo que es necesario buscar alternativas para el aprendizaje de los alumnos.

El equipo de investigación desarrolló, como parte central de la preparación del curso MOOC, de estructuras de datos varios objetos de aprendizaje que abordaron los tópicos relacionados al uso de los árboles binarios. La evaluación de los alumnos que los usaron fue favorable en los aspectos de: percepción de utilidad de los materiales, la pertinencia de las actividades, la facilidad de las tareas encomendadas y sobre la claridad de indicaciones y cuestionamientos. Los resultados de esta evaluación permiten considerar que los objetos de aprendizaje desarrollados en esta fase de trabajo serán bien recibidos en el curso MOOC del cual serán parte.

PALABRAS CLAVE recursos para el aprendizaje, programación de computadoras, estudio independiente.

\section{ABSTRACT}

This paper describes the development and evaluation of various learning objects that will be used as components of a MOOC (massive open online course) within the framework of the Digital Production Communities managed by the CODAES network (Comunidades Digitales para el Aprendizaje en Educación Superior) in Mexico. The materials arise from the need to provide higher-level students of computer-related occupations with different means of learning about complex and necessary subjects in their training. Current failure rates show that traditional teaching techniques have not been effective in achieving the learning of some topics such as programming; the data confirm that this competence is difficult for students to acquire and therefore it is necessary to seek learning alternatives. 
The research team developed as a central part of the preparation of the MOOC course on data structures, several learning objects that addressed the topics related to the use of binary trees. The evaluation of the students who used them was favorable in the aspects of perception of the usefulness of materials, the relevance of activities, the ease of tasks and the clarity of indications and questions. The evaluation results allow us to consider that the learning objects developed in this phase of work will be well received in the $\mathrm{MOOC}$ course in which they will be a part of.

KEYWORDS learning resources, computer programming, independent study.

\section{INTRODUCCIÓN}

En este trabajo se describe el desarrollo y evaluación de objetos de aprendizaje que se usarán como componentes de un curso MOOC (massive open online course) como estrategia para fortalecer el aprendizaje de las estructuras de datos de alumnos de ciencias de la computación de tres universidades mexicanas: la Universidad Veracruzana, la Universidad Autónoma del Estado de México y la Benemérita Universidad Autónoma de Puebla. El equipo de desarrollo forma parte de una comunidad digital de producción de la red Comunidades Digitales para el Aprendizaje en Educación Superior (CODAES).

El desarrollo de objetos de aprendizaje surge como respuesta a las problemáticas que deben resolverse en México: un país con más de 120 millones de habitantes que enfrenta grandes retos para brindar a su población los servicios que requiere y con la calidad que su población merece. Este reto es particularmente apremiante en el área de la educación y en el aprovechamiento de la tecnología.

El acceso a la educación de nivel superior es uno de los problemas que México no ha podido resolver. Cifras de la Organización para la Cooperación y Desarrollo Económico (OCDE) indican que, a pesar las mejoras alcanzadas en el año 2016 al lograr que el 25\% de jóvenes con edad de 25 a 34 años tengan la educación media superior concluida, solo el 17\% de las personas de entre 25 a 64 años de edad en México había cursado la educación superior en el mismo año; esto es la proporción más baja entre los países de la OCDE, 20 puntos porcentuales abajo del promedio de la OCDE (37\%).

Sin embargo, a pesar de esta situación, los datos de la OCDE reportan que México ha logrado una de las mayores proporciones de estudiantes que ingresan a la educación superior en el campo de la ciencia, tecnología, ingenierías y matemáticas entre los países miembros de la OCDE (OCDE, 2017). Por lo que es importante apoyar a este grupo de alumnos en su permanencia y tránsito por las instituciones de educación superior.

Aunado a los retos antes descritos, el país tiene un problema para aprovechar y usar la tecnología según el índice NRI (networked readiness index). De acuerdo con los reportes especializados México tiene el lugar 92 de 139 en el 2016 respecto a las habilidades que le permiten aprovechar la tecnología.

En este contexto se observa apremiante aplicar políticas públicas que faciliten el acceso de la población estudiantil a las TIC (tecnologías de informática y comunicaciones) e implementar estrategias que permitan a las universidades aprovechar el acceso a la web para ampliar sus servicios educativos, resolver problemas didácticos como el alto nivel de reprobación y así elevar la calidad de sus servicios.

\section{JUSTIFICACIÓN}

El impacto y uso que las TIC tienen en un país es representado por el NRI. Medido por el World Economic Forum, organización que entre otras actividades publica anualmente "The Global Information Technology 
Report”. En el documento se explica los criterios de evaluación y la metodología de medición, así como los resultados obtenidos por los países evaluados durante el año. Los resultados de México no han sido favorables durante los últimos cinco años; los reportes desde 2013 muestran que México no logra mejorar su nivel de aprovechamiento y disponibilidad de la tecnología, pues la ubicación lograda en el ranking mundial no logra superar el lugar 63, que ha sido el mejor nivel logrado en el año 2013. Los índices de México han fluctuado entre el lugar 63 y el 79, a pesar de los cambios en las políticas públicas y de las reformas que se han implementado en el país.

El índice general representa el puntaje obtenido a partir de 10 factores medibles a los que el World Economic Forum nombra pilares. Estos pilares se agrupan en cuatro subíndices que describen cuatro aspectos críticos en el uso y acceso de la tecnología: el entorno, la disponibilidad, el uso y el impacto.

Los datos del año 2016 muestran que el área de mayor oportunidad de mejora para México se presenta en el subíndice de disponibilidad; en el pilar de habilidades México logra el lugar 92 de 139 países. Este indicador valora la calidad del sistema educativo del país, la calidad en la educación de matemáticas y ciencias; la tasa de matriculación de alumnos en nivel secundaria y la tasa de alfabetización de los adul-

TABLA 1. Valores obtenidos por México en los pilares y subíndices del NRI del año 2016 (Baller et al., 2016)

\begin{tabular}{|c|c|c|}
\hline \multicolumn{3}{|c|}{ NETWORKED READINESS INDEX PARA MÉXICO } \\
\hline & Lugar (de 139) & Valor (1-7) \\
\hline Networked Readiness & 76 & 4.0 \\
\hline \multicolumn{3}{|l|}{ Index 2016 (76 de 139) } \\
\hline Networked Readiness Index & 69 & 4.0 \\
\hline \multicolumn{3}{|l|}{2015 (69 de 143) } \\
\hline Networked Readiness Index & 79 & 3.9 \\
\hline \multicolumn{3}{|l|}{2014 (79 de 148) } \\
\hline Networked Readiness Index & 63 & 3.9 \\
\hline \multicolumn{3}{|l|}{2013 (63 de 144) } \\
\hline A. Subíndice del entorno & 79 & 3.9 \\
\hline 1er pilar: entorno político y & 77 & 3.7 \\
\hline $2^{\circ}$ pilar: entorno empresarial & 83 & 4.1 \\
\hline \multicolumn{3}{|l|}{ e innovación } \\
\hline B. Subíndice de disponibilidad & 84 & 4.6 \\
\hline 3er pilar: Infraestructura & 84 & 3.7 \\
\hline $4^{\circ}$ pilar: Asequibilidad & 54 & 5.7 \\
\hline $5^{\circ}$ pilar: Habilidades & 92 & 4.5 \\
\hline C. Subíndice de uso & 74 & 3.8 \\
\hline $6^{\circ}$ pilar: uso individual & 84 & 3.6 \\
\hline $7^{\circ}$ pilar: uso comercial & 66 & 3.6 \\
\hline D. Subíndice de impacto & 70 & 3.7 \\
\hline $8^{\circ}$ pilar: uso del gobierno & 50 & 4.2 \\
\hline $9^{\circ}$ pilar: impactos económicos & 64 & 3.3 \\
\hline $10^{\circ}$ pilar: impactos sociales & 71 & 4.1 \\
\hline
\end{tabular}
tos. Otro indicador por debajo del promedio de los países de altos recursos se refiere al pilar de la asequibilidad, en el indicador de ajuste a las tarifas de internet de banda ancha; en este factor México logró el lugar 94 de 139 países, lo que indica que, a pesar de la baja en los costos del Internet en México, aún el costo es poco asequible para la población. En la tabla 1 se observa con mayor detalle las puntuaciones obtenidas por México en el año 2016; obsérvese que el valor más favorable se refiere al pilar de uso del gobierno, índice que se ha incrementado continuamente y ha facilitado la comunicación del gobierno con la población; le sigue el puntaje de asequibilidad e impactos económicos.

En la figura 1 se observa la relación de los puntajes de México con el promedio del grupo de países con ingresos medios-altos. Se observa que los principales retos están en los pilares de asequibilidad, uso individual y habilidades. Los pilares en que México iguala al promedio son impacto social y uso comercial. Finalmente se puede observar que en los pilares de uso del gobierno y asequibilidad se supera por poco al promedio.

Por otro lado, los retos que enfrentan los alumnos de licenciaturas que estudian a la computación son variados, pero sobresale el problema del alto nivel de reprobación en asignaturas relacionadas con las matemáticas y la programación de computadoras. 
Estos indicadores inciden negativamente en la recepción de apoyos que los alumnos pueden recibir para mejorar su nivel académico, como las becas escolares o becas de apoyo para movilidades nacionales e internacionales. Con esta situación disminuye el nivel de inserción de los alumnos en estudios de posgrado en detrimento de las universidades y de las oportunidades de desarrollo de los mismos estudiantes.

Como ejemplo de esta situación se muestran los datos estadísticos de las unidades de aprendizaje con mayor nivel de reprobación en Ingeniería en Computación del Centro Universitario UAEM Texcoco.

En la tabla 2 y en la gráfica 1 se observa que los niveles de reprobación en la evaluación ordinaria son muy elevados en algunas unidades de aprendizaje, por ejemplo, Algebra lineal con el 60\% o Autómatas y lenguajes formales con el 55.7\%. Aunque algunos alumnos logran aprobar en evaluaciones extraordinarias o a título de suficiencia, los indicadores en su trayectoria los señalan como alumnos irregulares y, por esta situación, pierden el derecho a participar en convocatorias para obtener apoyos institucionales, estatales o federales orientados a propiciar la permanecía de los alumnos y apoyar la retención estudiantil.

Este problema no es exclusivo de la UAEM, los valores de la tabla se acercan a los documentados por varios autores que han tratado este problema en la enseñanza de las ingenierías (Avalos et al. 2014; Guzmán Valdivia, 2013).

TABLA 2. Unidades de aprendizaje de mayor reprobación en Ingeniería en computación del CU UAEM Texcoco (Control Escolar UAEM)

\begin{tabular}{|c|c|c|c|c|}
\hline & UNIDAD DE APRENDIZAJE & ALUMNOS INSCRITOS & ALUMNOS REPROBADOS & ÍNDICE DE REPROBACIÓN \\
\hline \multirow{5}{*}{ 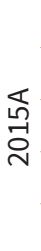 } & Calculo 2 & 63.00 & 116.00 & 54.3 \\
\hline & Estructuras de datos & 66.00 & 123.00 & 53.7 \\
\hline & Autómatas y lenguajes formales & 73.00 & 131.00 & 55.7 \\
\hline & Administración de recursos informáticos & 77.00 & 227.00 & 33.9 \\
\hline & Algebra lineal & 78.00 & 130.00 & 60.0 \\
\hline \multirow{5}{*}{$\begin{array}{l}\widetilde{0} \\
-1 \\
\text { ○్ }\end{array}$} & Estructuras de datos & 56.00 & 130.00 & 43.1 \\
\hline & Lógica secuencial y combinatoria & 58.00 & 100.00 & 58.0 \\
\hline & Fundamentos de bases de datos & 66.00 & 122.00 & 54.1 \\
\hline & Probabilidad y estadística & 71.00 & 128.00 & 55.5 \\
\hline & Sistemas operativos & 74.00 & 164.00 & 45.1 \\
\hline \multirow{5}{*}{$\underset{\mathbb{N}}{\stackrel{\mathbb{N}}{O}}$} & Física básica & 73.00 & 150.00 & 48.7 \\
\hline & Estructuras de datos & 78.00 & 136.00 & 57.4 \\
\hline & Probabilidad y estadística & 79.00 & 153.00 & 51.6 \\
\hline & Autómatas y lenguajes formales & 91.00 & 137.00 & 66.4 \\
\hline & Álgebra lineal & 101.00 & 171.00 & 59.1 \\
\hline
\end{tabular}
respecto al promedio del grupo de países con ingresos medios-altos (World Economic Forum, 2016)

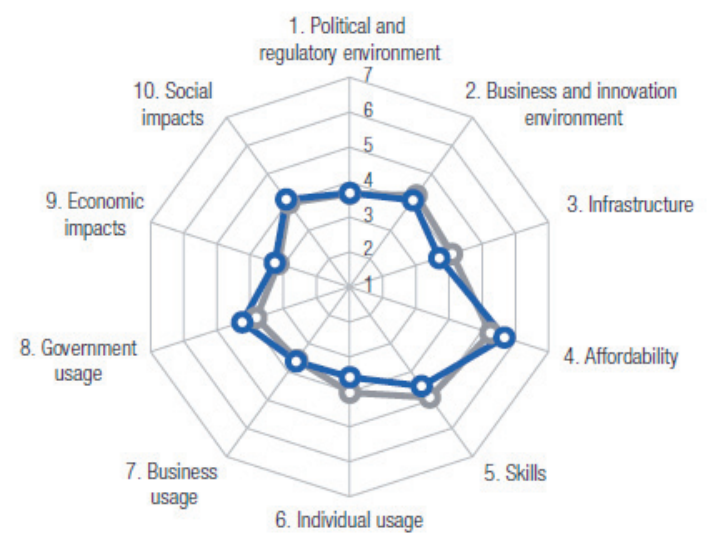

- Mexico $\quad$-O=- Upper-middle-income group average
Figura 1. Relación de los valores obtenidos por México 
GRÁFICA 1. Unidades de aprendizaje de mayor reprobación en Ingeniería en Computación del CU UAEM Texcoco

Índice de reprobación de las Unidades de aprendizaje de mayor reprobación

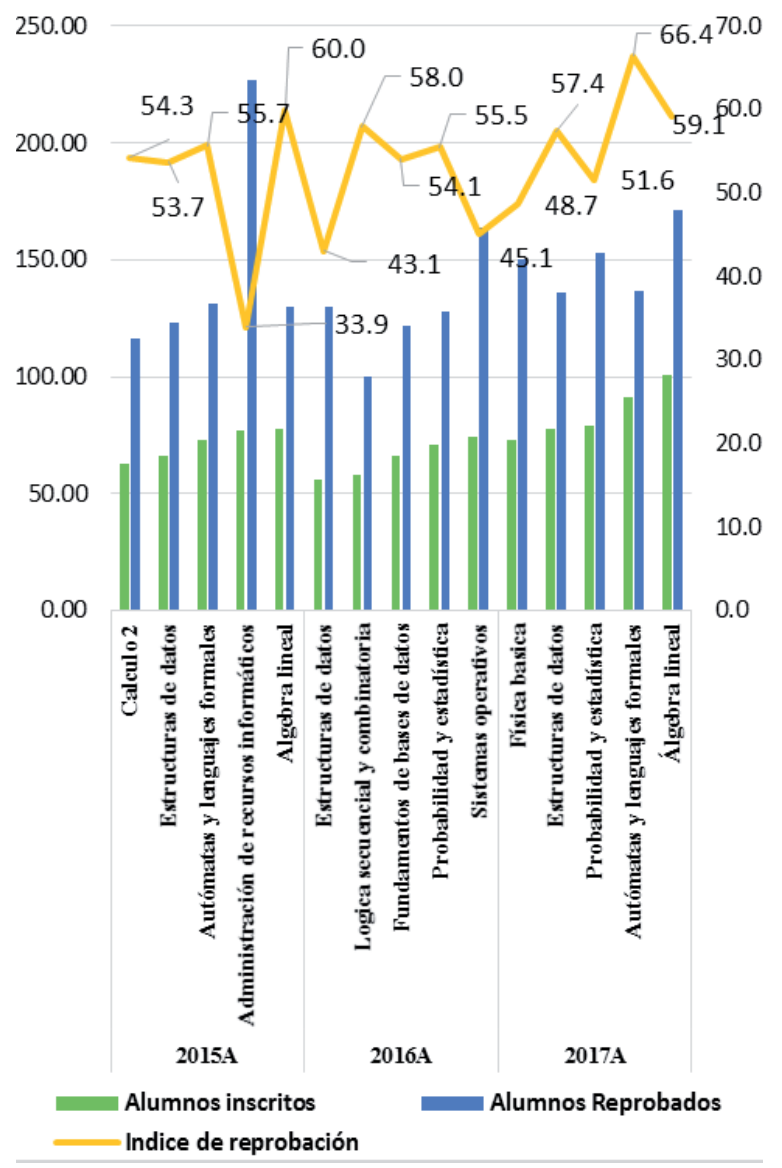

La situación de la UAEM coincide con los argumentos expresados por Urbina Nájera (2019) respecto a que, para las carreras de ingeniería en informática o análogas, los altos índices de reprobación suceden con materias como programación, ingeniería de software, análisis de sistemas, diseño de sistemas, además de las propias del área de formación.

Para abordar este problema, usando y aprovechando los beneficios de las nuevas tecnologías de la informática, se han implementado varias estrategias, por ejemplo, el uso del aprendizaje hibrido (presencial y en línea) mediante la implementación de cursos MOOC (Jiménez Cruz, 2017).

En la comunidad de especialistas se está explorando el uso de juegos serios (JS) para facilitar el aprendizaje de los alumnos; al respecto, los estudios más recientes muestran una mejora de aprendizaje con el uso de JS comparados con los métodos tradicionales (García-Mundo L y otros 2019).

Aunque los juegos serios también presentan algunas dificultades, sobre todo en la evaluación, también es importante en su efectividad la selección adecuada de los juegos serios para el dominio requerido, la capacidad del JS de generar compromiso sin olvidar que la finalidad del juego serio es promover la motivación y el aprendizaje del alumno (García-Mundo et al., 2019).

También se está explorando un enfoque basado en la visualización mediante software, ya que la enseñanza de la programación y la manipulación de estructuras de datos encuentran, con la visualización de software, un mecanismo de enseñanza y aprendizaje que ayuda fundamentalmente a la lectura comprensiva de algoritmos (González et al., 2004).

La exploración del aprendizaje mediante actividades lúdicas, desarrollada por Astete (2014), mediante los videojuegos procura apoyar el desarrollo de competencias TIC. Su propuesta presenta una innovadora forma de promover el aprendizaje en la educación, implementando un curso de formación docente a través de un videojuego, aplicando recursos educativos digitales e impulsando la competencia tecnología en los alumnos, competencias que hoy necesitan en su formación profesional.

Otro intento por desarrollar nuevas formas de enseñar, específicamente a programar, es el caso de López-Escribano (2012), quien explora el uso de Scratch como medio de facilitar la compresión de la programación con niños con diferentes necesidades y estilos de aprendizaje. Ofrece una lista de recursos para hacer el programa accesible, pudiendo aprender este lenguaje de programación. Aunque Scratch está dedicado en desarrollar juegos computacionales bajo su misma plataforma puede enseñar y desarrollar la lógica de programación desde edades tempranas. 
En México se implementan propuestas gubernamentales para apoyar las iniciativas de las universidades interesadas en abordar estos problemas. Uno de los resultados de estos apoyos ha sido el surgimiento de la red CODAES, una comunidad formada por diversos grupos de expertos dedicados al desarrollo de aplicaciones y de recursos educativos, que son concebidos como herramientas de apoyo a los procesos de enseñanza-aprendizaje en la educación superior, fomentan la innovación educativa, el desarrollo de formadores, la actualización docente y la vinculación de la universidad con la sociedad (Red CODAES, 2014).

\subsection{Los beneficios de los OA (objetos de aprendizaje)}

Los objetos de aprendizaje son unidades de material didáctico que tienen la finalidad de ser reutilizables en diversas secuencias de aprendizaje, se caracterizan por usar estándares para ser definidos de acuerdo con su temática, formato de archivo, nivel de aprendizaje entre otras características, de tal forma que se facilite su almacenamiento y recuperación en los repositorios de objetos de aprendizaje.

Se recomienda que los objetos de aprendizaje sean adaptables para satisfacer las expectativas y necesidades de los alumnos y docentes, de esta manera se incrementa el interés por el material didáctico. Se debe procurar que el material sea fácilmente entendible y con buen nivel de usabilidad para lograr una mejor comprensión del usuario que interactúa con el material.

Con ayuda de los recursos digitales se puede implementar un aprendizaje híbrido, el cual consiste en tomar clases como se hace normalmente y también llevar a cabo actividades en línea. La finalidad es que con esta combinación los alumnos tengan las herramientas necesarias para reforzar lo aprendido en clase y tener una mejor comprensión de los temas del curso (Jimenez Cruz, 2017).

El aprendizaje híbrido tiene la gran ventaja de que hoy en día los medios multimedia están al alcance de todo el mundo y los alumnos están muy familiarizados con su uso y reusó en el momento que se necesiten.

Entre las ventajas de usar el aprendizaje híbrido mediante OA se identifican:

- Ayuda a través de la multimedia a transmitir contenido didáctico.

- Permiten implementar formas de educación distintas a las tradicionales y a enriquecer las experiencias de aprendizaje.

- Se usan y aprovechan los formatos más amigables: fotos, vídeos, juegos serios, etc.

- Facilita que el estudiante ponga a prueba los conocimientos adquiridos en actividades que les causan interés.

- Permiten consultas y repaso más allá del espacio físico y temporal de las clases en el aula.

- Permiten al alumno gestionar su propio aprendizaje.

- Apoyan al docente en la enseñanza y la enriquece, aumentando la aceptación en los alumnos.

- Permiten que los estudiantes tomen un papel más activo en el aprendizaje.

\subsection{La importancia de las estructuras de datos en la computación}

La gran utilidad de las computadoras radica en la alta capacidad que tienen estos dispositivos para procesar información. Gracias a esto son capaces de realizar actividades de forma eficiente y automatizada, pero para que esta información pueda ser procesada se requiere que esté almacenada en la memoria de la computadora. 
Para almacenar información en memoria los datos se clasifican en dos formas:

- Tipos de datos simples.

- Tipos de datos estructurados.

Los tipos de datos simples tienen la característica de ocupar un solo espacio en memoria, esto nos indica que una variable simple hace referencia a un único valor, a diferencia de los tipos de datos estructurados, que se caracterizan por utilizar un solo nombre para hacer referencia a un conjunto de componentes, donde cada uno puede ser un tipo de dato simple o estructurado.

En el desarrollo de software los tipos de datos estructurados también son llamadas estructuras de datos estáticas, y son de gran utilidad para almacenar conjuntos de datos, pero tienen la limitación de que el espacio ocupado en memoria se define en tiempo de compilación y no puede ser cambiado durante la ejecución del programa. Para solucionar este problema existen las estructuras de datos dinámicas como son las pilas, colas, listas, árboles, etc., que pueden almacenar grandes cantidades de elementos de diferentes tipos (Arias Guerra y Arias Guerra, 2011)

Para el desarrollo de software es indispensable conocer el funcionamiento de las estructuras de datos; por lo tanto, y debido a su importancia, es prácticamente obligatorio el estudio de las estructuras de datos en su versión más simple (listas, pilas, colas, árboles y grafos sencillos), lo cual tiene la finalidad de utilizar dichas estructuras de datos para dar solución a determinados problemas, principalmente los que requieran almacenar conjuntos grandes de datos en memoria.

Olabe (2015) recomienda desarrollar el pensamiento computacional y cómo puede ser agregado en clases a través del diseño e implementación de los proyectos de programación. Se menciona las necesidades, el propósito y la característica más importante del pensamiento computacional. Muestra ejemplos con elementos fundamentales del pensamiento computacional utilizando lenguajes de programación. Muestra los resultados del proyecto del pensamiento computacional en la escuela.

\section{MATERIAL Y MÉTODOS}

\subsection{Diseño didáctico}

De acuerdo con las recomendaciones de la Red CODAES (2015) el documento base que sirve para especificar el diseño instruccional es el guion instruccional, que permite al equipo de desarrollo describir y especificar todas las características de los contenidos y su recomendación de uso para lograr los objetivos educativos de los materiales. Para que el guion instruccional sea eficiente en el proceso de desarrollo se recomienda que cumpla con las siguientes características:

- Debe indicar la presentación de los contenidos organizados en la pantalla.

- Debe responder a las necesidades del diseñador instruccional, diseñador gráfico y del programador.

- Debe incluir tres elementos básicos; los contenidos, el diseño gráfico y la programación de las animaciones.

- Debe mostrar las estructuras del contenido y sus jerarquías.

- Debe ser suficientemente claro y especifico.

- Debe adaptarse a las características del proyecto y de su contexto educativo y grupo de usuarios. 
Para especificar el diseño didáctico, en este trabajo se usó un formato de secuencia didáctica para cada objeto de aprendizaje. En él se describen los principales puntos que guían didácticamente el uso de los objetos de aprendizaje y, además, pueden usarse como base para definir los metadatos del objeto de aprendizaje. También se usa el guion multimedia para describir las características detalladas gráficas y de interacción del objeto a desarrollar. A continuación, en la tabla 3 se muestra un ejemplo de dicho formato.

TABLA 3. Secuencia didáctica para objetos de aprendizaje

\begin{tabular}{llll}
\hline & \multicolumn{2}{c}{ ACTIVIDAD DE APRENDIZAJE } \\
\hline Objeto de aprendizaje & $\begin{array}{l}\text { Recorrido preorden de un } \\
\text { árbol binario }\end{array}$ & Granularidad & 2 \\
\hline \multirow{2}{*}{ Objeto de aprendizaje antecedente } & $\begin{array}{l}\text { Conceptos básicos de } \\
\text { árbol binario }\end{array}$ & $\begin{array}{l}\text { Objeto de aprendizaje } \\
\text { precedente }\end{array}$ & Recorrido inorden de un árbol bi- \\
\hline Tema General & Árboles binarios & Tema específico & Recorridos de árboles binarios \\
\hline Autor & Equipo UAEM & Guion multimedia & Guion 4 \\
\hline
\end{tabular}

Jerarquía conceptual

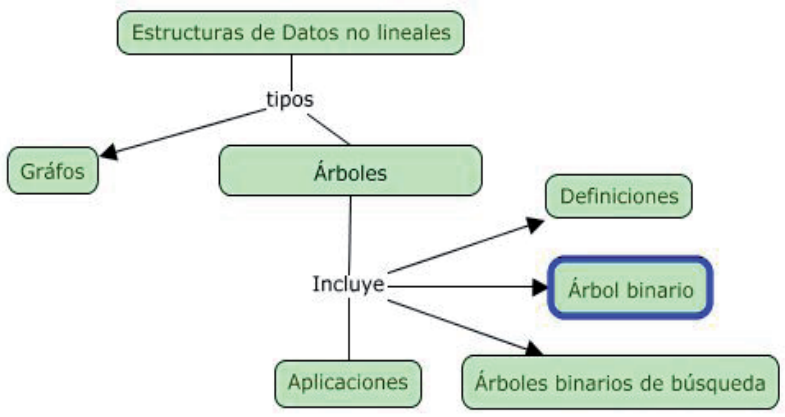

\begin{tabular}{ll}
\hline Intensión formativa & $\begin{array}{l}\text { El alumno comprenderá el algoritmo recursivo preorden como medio de conocer los elemen- } \\
\text { tos almacenados en un árbol binario }\end{array}$ \\
\hline Contenido Conceptual & Conceptos: recorrido recursivo, consulta de nodos, uso de funciones recursivas \\
\hline Objetivo Procedimental & $\begin{array}{l}\text { El alumno usará las reglas del algoritmo preorden para consultar los elementos almacenados } \\
\text { en un árbol binario }\end{array}$ \\
\hline Objetivo Actitudinal & $\begin{array}{l}\text { El alumno desarrollará su capacidad de ser atento, responsable y constante en el desarrollo } \\
\text { de sus actividades }\end{array}$ \\
\hline Tipo de Actividad de Aprendizaje & $\begin{array}{l}\text { () Apertura (X) Desarrollo () Cierre } \\
\text { () Todas }\end{array}$ \\
\hline Actividades para el alumno & $\begin{array}{l}\text { El alumno lee y escucha la explicación sobre el recorrido preorden de un video animado; el } \\
\text { alumno responde cuestionamientos sobre la aplicación del algoritmo en varios árboles bi- } \\
\text { narios; para responder el alumno ejecuta el algoritmo y recibe retroalimentación del objeto } \\
\text { de aprendizaje }\end{array}$ \\
\hline Actividades del alumno &
\end{tabular}

Actividades en grupo

En el objeto de aprendizaje ninguna, pero puede usarse en la clase presencial para aplicar diversas dinámicas de grupo como competencias por equipos, por ejemplo.

Evaluación o autoevaluación

(X) cuestionario () redacción de texto

() entrega de productos ( ) actividades lúdicas

Puntaje o calificación

Variable de acuerdo con los docentes y a su uso 


\begin{tabular}{ll}
\hline Aspectos Gráficos & \\
\hline Tipo de letra & Sans-serif \\
\hline Color de títulos & $98 \mathrm{~b} 239 \mathrm{ff}$ \\
\hline Diámetro de nodo raíz & $17.6 \mathrm{~mm}$ \\
\hline Diámetro de nodo intermedio & $16.0 \mathrm{~mm}$ \\
\hline Grosor de arista & $0.5 \mathrm{~mm}$ \\
\hline Color de rama & $168383 \mathrm{ff}$ \\
\hline Color de letra nodo intermedio & $00007 \mathrm{aff}$ \\
\hline Color de letra nodo terminal & $004 \mathrm{e} 00 \mathrm{ff}$ \\
\hline Color nodo intermedio azul & $55695 \mathrm{eff}$ \\
\hline Color nodo terminal verde & $84 \mathrm{c} 822 \mathrm{ff}$
\end{tabular}

FIGURA 2. Definición de aspectos gráficos para la elaboración de árboles

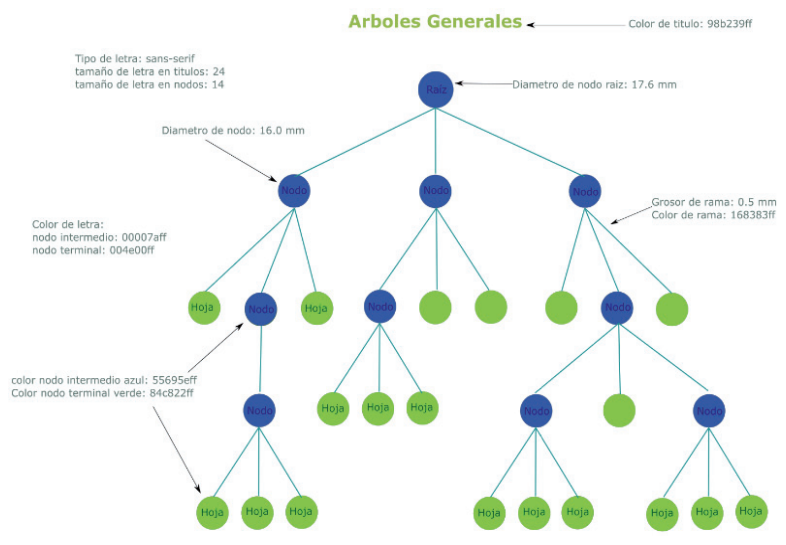

\subsection{Descripción de la población}

El estudio se desarrolló en el Centro Universitario UAEM Texcoco, durante el semestre 2018A con alumnos de segundo semestre que en ese periodo cursaron estructura de datos. Los OA fueron distribuidos por el grupo de desarrollo durante el primer periodo de evaluación parcial, a la mitad del curso; antes de usarlos se aplicó a los alumnos un pequeño cuestionario para valorar el conocimiento que hasta ese momento tenían de las estructuras de datos básicas. Posteriormente se les entregaron los OA y se les dieron dos semanas para que los usaran en su estudio independiente sobre su materia. El uso de

los OA por el alumnado se promovió como material de estudio complementario a sugerencia de los profesores; su uso no fue impuesto ni monitoreado por el profesor, sino que se dejó a la libre decisión del alumno.

Pasadas tres semanas de haber distribuido los OA se aplicó a los alumnos un segundo cuestionario para valorar cambios en el dominio de los temas evaluados y para conocer la opinión respecto a utilidad de los materiales.

Al analizar los datos obtenidos se observó una situación que no se contempló en el diseño del estudio: la participación de los alumnos no fue constante en número pues se incrementó a través del estudio, ya que con la recomendación entre compañeros el número de alumnos se incrementó de 53 a 87. La respuesta de los alumnos al segundo cuestionario, como puede observarse en la tabla 4, se incrementó del 43\% de los invitados al $65.9 \%$ en la segunda fase del estudio.

\subsection{Los instrumentos}

Se aplicaron dos cuestionarios que evaluaron el conocimiento de los conceptos básicos y manejo de estructuras de datos sobre dos unidades, la primera que trató los conceptos y conocimientos generales referentes a las estructuras de datos básicas (pilas, colas y listas), mientras que la segunda unidad se centra en conocimientos más avanzados para evaluar el manejo de árboles binarios. 
TABLA 4. Participación de los estudiantes en el estudio

\begin{tabular}{lcc}
\hline & & PORCENTAJE \\
\hline Alumnos inscritos en estructuras de datos & 158 & $100 \%$ \\
\hline Alumnos invitados a usar objetos de aprendizaje (tienen asistencia regular) & 132 & $83 \%$ de los inscritos \\
\hline Alumnos que recibieron los objetos de aprendizaje & 132 & $100 \%$ de los invitados \\
\hline Alumnos que respondieron el primer cuestionario & 57 & $43 \%$ de los invitados \\
\hline Alumnos que respondieron el segundo cuestionario & 87 & $65.9 \%$ de los invitados \\
\hline
\end{tabular}

Los cuestionarios se formaron con 31 reactivos de opción múltiple:

1. ¿Qué es una estructura lineal?

2. FIFO (First Input, First Output) es una característica de un tipo de estructura lineal, ¿cuál es la estructura a la que pertenece?

3. LIFO (Last Input, First Output) es una característica de un tipo de estructura lineal, ¿cuál es la estructura a la que pertenece?

4. ¿En qué consiste una estructura dinámica?

5. ¿Qué es un nodo?

6. ¿Cómo se define una lista ordenada?

7. ¿Qué es un apuntador?

8. Las operaciones push y pop pertenecen a una de las siguientes estructuras, ¿cuál es la estructura a la que pertenece?

9. ¿Para qué sirve el tope de pila?

10. Si se considera que la siguiente imagen es la estructura de una pila, ¿qué elemento debe corresponder al tope de pila?

11. ¿Cuál de las siguientes expresiones es una característica de una cola?

12. ¿Cuál de las siguientes expresiones es una característica de una pila?

13. ¿Por qué es importante usar apuntadores en la implementación de una estructura dinámica?

14. ¿Cómo se realiza el borrado de un nodo en una lista ordenada?

15. ¿Cuáles de las siguientes afirmaciones pertenecen a ejemplos de pilas?

16. ¿Cuáles de las siguientes afirmaciones pertenecen a ejemplos de colas?

17. En estructura de datos, ¿qué es un árbol binario?

18. ¿Cuál es el tipo de estructura al que pertenecen los árboles?

19. ¿Cuál es el nodo más importante de un árbol?

20. ¿Cómo se define una hoja?

21. ¿Cuáles son las tres formas de recorrer un árbol?

22. ¿Cuál es la definición de longitud de camino?

23. ¿Qué es una arista? 
24. ¿Cuál es la forma que sigue el recorrido en preorden?

25. ¿Qué característica deben tener dos nodos para decirse que son hermanos?

26. ¿Qué diferencia existe entre un árbol general y un árbol binario?

27. El recorrido del siguiente árbol (imagen) da como resultado 3, 6, 5, 21, 22, 10, 16, 28, 27, 32, 33, 30 , 25. ¿Qué tipo de recorrido fue el que se realizó?

28. Además de los árboles, menciona otro tipo de estructuras no lineales.

29. ¿Cuál es el grado de un árbol binario?

30. ¿Cuáles el resultado de recorrer el siguiente árbol binario en preorden?

31. ¿Cuál es la altura del siguiente árbol?

El segundo cuestionario se aplicó para evaluar el desempeño de los alumnos al término de haber estudiado con los OA con el fin de evaluar la diferencia en el manejo de los conceptos al finalizar el proceso; el cuestionario fue el mismo que el primero, solo que se añadió una sección para valorar la apreciación del material, con la finalidad de tener en cuenta la opinión de los alumnos para acciones de mejora en nuevos objetos de aprendizaje.

Las preguntas que se agregaron al segundo cuestionario son las siguientes:

- En escala de 1 a 5, ¿qué tan pertinentes considera que son las actividades del objeto de aprendizaje?

- En escala de 1 a 5, ¿qué tan correcto considera que fue el aspecto didáctico en los objetos de aprendizaje?

- ¿Qué tan fácil considera que fueron las tareas en los objetos de aprendizaje?

- ¿Considera que realizar las actividades del objeto de aprendizaje le ayudaron a comprender mejor el tema tratado?

- ¿Las preguntas de evaluación fueron bien redactadas?

- ¿Las explicaciones de los temas fueron claros?

- ¿Los vídeos le parecieron útiles?

- Si pudiera agregar temas al objeto de aprendizaje, ¿cuáles agregaría?

- Si hubiera la posibilidad de tener más objetos de aprendizaje, ¿qué tema le interesaría trabajar con ellos?

En la escala de valores el numero 1 significa malos, inconvenientes o complicados y el número 5 significa excelentes, muy pertinentes o fáciles, según la pregunta.

\section{RESULTADOS}

La participación de los alumnos fue distinta en las dos fases de aplicación del cuestionario, por lo que se muestran los resultados en términos de porcentaje. La gráfica 2 muestra los resultados del primer cuestionario y la 3 las del segundo cuestionario, siendo un total de 31 preguntas.

En la gráfica 2 se muestra el conteo de respuestas correctas, incorrectas y no respondidas por los alumnos. 
Analizando las respuestas con respecto a cada pregunta se observa que, en problemas de aplicación como las preguntas de la 23 a la 28, el número de aciertos de los alumnos en general disminuye; en estas preguntas en las que se debe responder a definiciones técnicas para resolver problemas reales, como por ejemplo ¿la forma de recorrer un árbol en preorden?, o ¿cómo se debe calcular el grado de un árbol general o binario? Los alumnos tienen bajo desempeño.

Esto puede ser debido a que los alumnos frecuentemente confunden los procesos, además de que puede deberse a la falta de práctica con ejercicios que reflejen el proceso funcional de las operaciones. Hay que tener en cuenta que un proceso se memoriza después de practicarlo varias veces.

Por otro lado, en los datos históricos de las calificaciones de los alumnos en la materia de estructura de datos respecto a los dos años anteriores, se observa que durante el semestre 2018A se presentó un ligero incremento en el promedio ,y aunque los factores de este incremento pueden ser de diferente naturaleza,

Gráfico 2. Resultado de la evaluación de los alumnos de estructura de datos antes de usar los OA

\section{Res puestas al cue stionario 1 R Incorrecta $\quad$ R correcta}

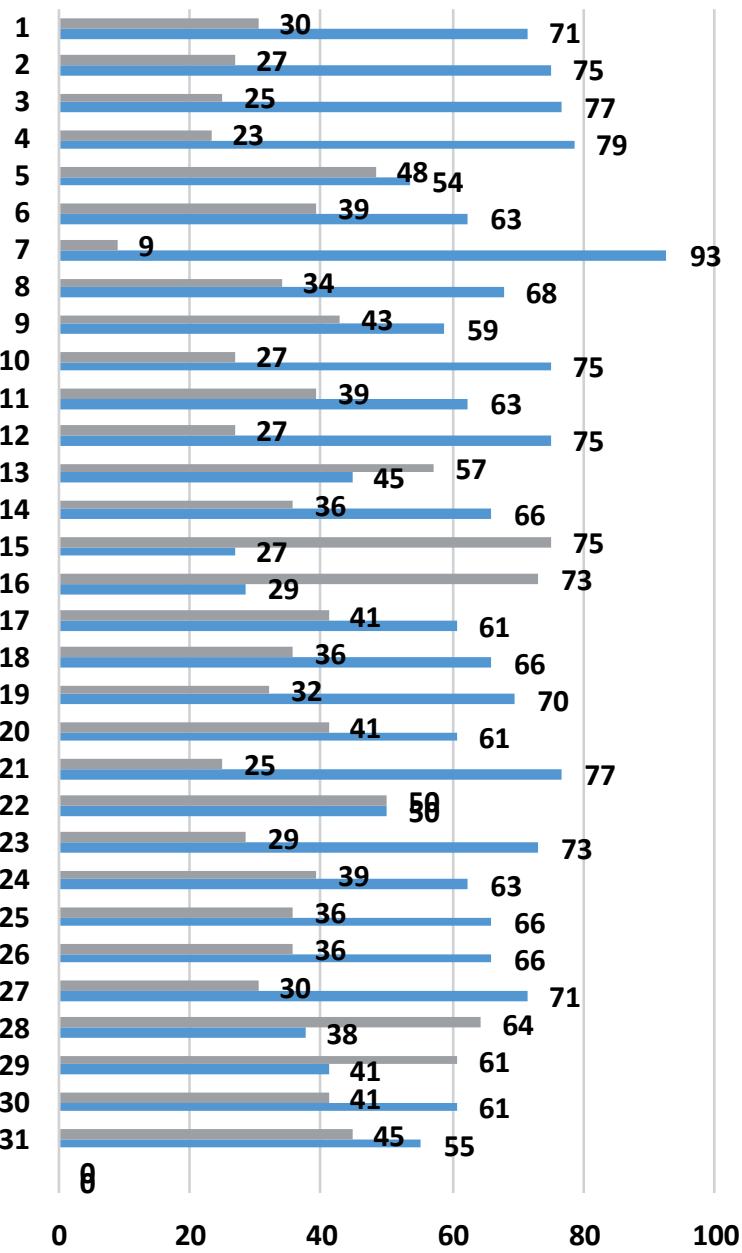

Gráfico 3. Resultado de la evaluación de los alumnos de estructura de datos después de usar OA

Respuestas del cuestionario 2 $\square$ Sin respuesta $\square \mathbf{2} \mathbf{R}$ incorrecta $\square \mathbf{2} \mathbf{R}$ Correcta

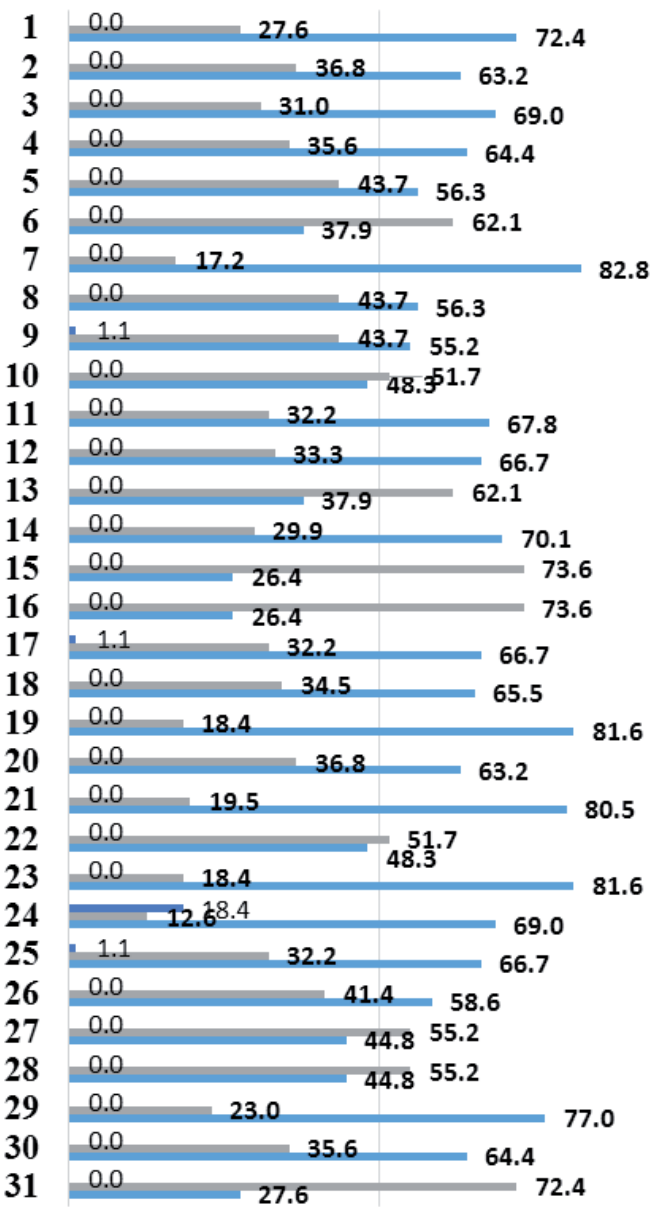

0.0

50.0

100.0 
el uso de los $\mathrm{OA}$ son un elemento que debe incluirse como factor de cambio pues el resto de las condiciones se presentaron constantes.

\subsection{La opinión de los alumnos encuestados sobre los OA}

La segunda sección del cuestionario fue realizada con la premisa de evaluar la utilidad de los $\mathrm{OA}$ distribuidos entre los alumnos para que, de esta forma, se pueda tener una mejor referencia de la utilidad que presentan los OA dentro de la comunidad universitaria y de la carrera de ingeniería en computación.

La opinión de los alumnos respecto a los OA se denota favorable (gráfico 5). Se observa la proporción de alumnos que consideran que les fue de utilidad los $O A$ y estos les sirvieron para comprender mejor los temas estudiados.

En el gráfico se puede mostrar que aproximadamente el 50\% de la población evaluada consideran que les fueron de gran utilidad los objetos de aprendizaje para comprender mejor los temas de estructura de datos, además de que les ayudaron a reforzar sus conocimientos básicos.

Por otro lado, el 31\% de la población aproximadamente respondió que estos solo les sirvieron para reforzar algunos conceptos generales de los temas, mientras que solo el $6.7 \%$ consideró que los $\mathrm{OA}$ no les fueron útiles para comprender ni para reforzar los temas de estructura de datos.

Respecto a la pertinencia de las actividades del $\mathrm{OA}$, las respuestas de los alumnos se muestran en el gráfico 6 (escala de 1 a 5: 1-malos, 5-excelentes). Las calificaciones con mayor porcentaje son 4 y 5 , que corresponden a las calificaciones más altas y significa que están de acuerdo en que las actividades realizadas fueron pertinentes.

Con respecto al resultado de la pregunta ¿Qué tan correcto considera que fue el aspecto didáctico en los objetos de aprendizaje? (1-incorrecto, 5-muy correcto), en el gráfico 7 se muestra que los porcentajes más altos corresponden a las calificaciones 4 y 5 , sumando un porcentaje de $61 \%$ siendo la mayor parte de la
Gráfico 4. Datos históricos del promedio de los alumnos en Estructura de datos

\section{Promedio de calificaciones de estructuras de datos}

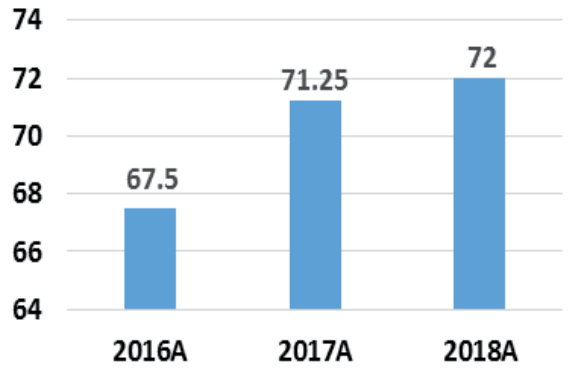

Gráfico 5. Resultado sobre la utilidad de los $\mathrm{OA}$

\section{Porcentaje de alumnos que consideran que los $\mathrm{OA}$ les ayudaron a comprender mejor los temas tratados.}

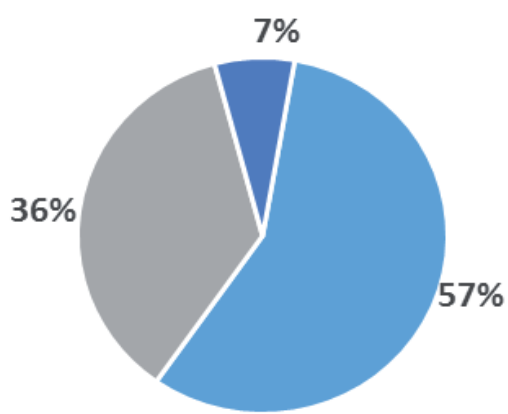

- Me fueron muy utiles para comprender los temas

- Solo me fueron utiles para aprobar aclarar ciertas dudas

- No me fuerton nada utiles

Gráfico 6. Resultado sobre la pertinencia de los OA

\section{Porcentaje de respuestas ¿Quétan pertinentes considera que son las actividades del objeto de aprendizaje?}

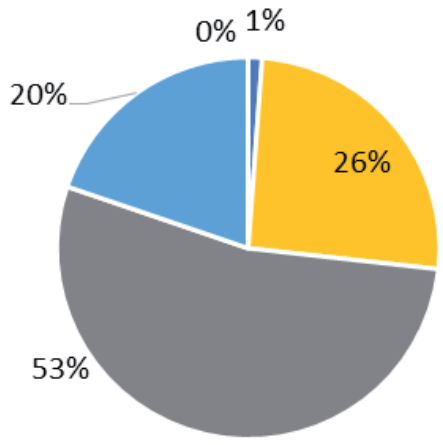

- Frec Opinión 1

- Frec Opinión 2

* Frec Opinión 3

- Frec Opinión 4

॥ Frec Opinión 5 
población concluimos que los aspectos didácticos incluidos en los OA son aprobados por los alumnos.

La pregunta ¿Qué tan fácil considera que fueron las tareas en los objetos de aprendizaje? (1-complicado; 5-muy fáciles) pretende evaluar la dificultad que tuvieron los alumnos para manejar e interactuar con los objetos de aprendizaje. Como se muestra en el gráfico 8 la calificación con mayor porcentaje de votantes corresponde a la calificación 3 y 4, lo cual indica que los alumnos consideran que las tareas realizadas tuvieron un grado de dificultad moderado y que se considera aceptable puesto que, al tratarse de tareas que desempeñan los alumnos, se integra cierto grado de dificultad para reforzar los conocimientos de los alumnos.

En la pregunta "LLas preguntas de evaluación fueron bien redactadas? ", se requiere evaluar si las preguntas fueron redactadas de una forma correcta y entendible para los alumnos, en el gráfico 9 se aprecia que el $87 \%$ de la población evaluada considera que las preguntas de evaluación fueron redactadas de forma correcta y entendible.

La pregunta "¿Las explicaciones de los temas fueron claros?" pretende conocer la opinión de los alumnos con respecto la forma de explicar los temas expuestos, al ver el gráfico 10 es notable que el $77 \%$ de la población considera que las explicaciones fueron claras y concisas, sin embargo, queda margen de mejora en esta sección.

En los resultados sobre la pregunta "¿Los vídeos le parecieron útiles?" se puede apreciar en el gráfico 11 que al $43 \%$ de la población le fueron útiles los vídeos en algún sentido general o particular, mientras que el $34 \%$ no están seguros si en verdad les fueron útiles y solo al $23 \%$ están convencidos que no les fueron útiles en lo más mínimo. Por tanto, se considera que lo más conveniente sería incluir más vídeos o explicar con mayor detalle la información.

En el gráfico 12 se muestran los resultados obtenidos de la pregunta ¿cuáles son los temas de su interés que les serían de utilidad como OA? Para realizar esta pregunta se tomaron en cuenta las materias con mayor índice de reprobación según datos de control escolar.
Gráfico 7. Resultado de la pregunta sobre el aspecto didáctico de los $\mathrm{OA}$

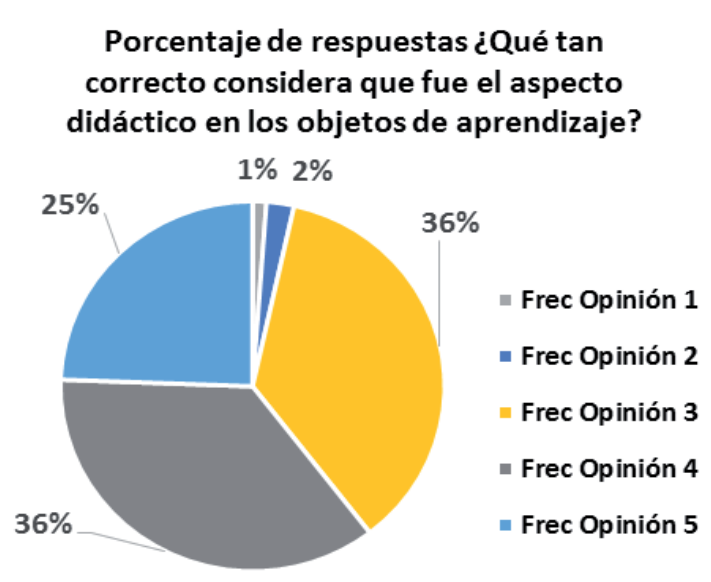

Gráfico 8. Resultado sobre la facilidad de las tareas en los OA

\section{Porcentaje de respuestas ¿Qué tan fácil considera que fueron las tareas en los objetos de aprendizaje?}

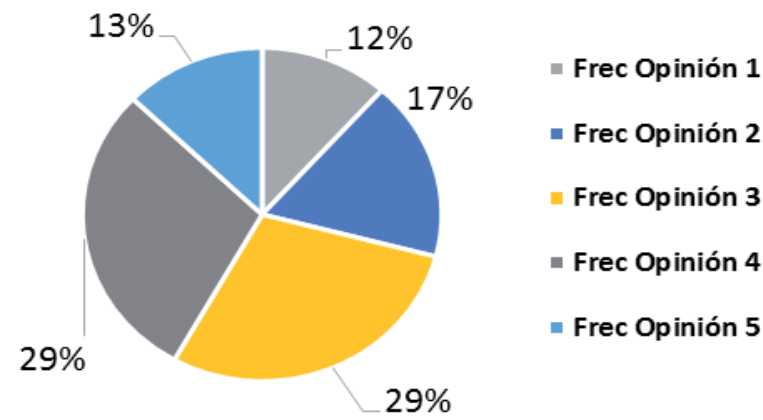

Gráfico 9. Resultados sobre la redacción de las preguntas

\section{¿Las preguntas de evaluación fueron bien redactadas?}

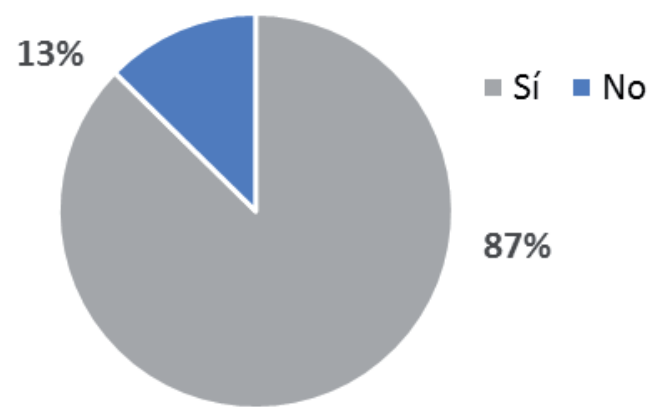


Un punto importante a considerar en esta gráfica es que se destaca la preferencia de los alumnos por OA que les ayuden a comprender y mejorar en materias relacionadas con programación, siendo esta una de las áreas que más se les complica entender.

\section{DISCUSIÓN}

Los resultados de la evaluación preliminar de los OA generados en este proyecto de investigación son una fuente de datos que retroalimenta el proceso de desarrollo. Con esta información el equipo puede ajustar los OA en puntos específicos como las explicaciones o la redacción de los cuestionamientos. Esto nos ayuda a elevar la eficiencia comunicativa de estos materiales y, en consecuencia, el nivel de eficiencia del curso masivo del cual serán parte.

El diseño didáctico basado en las recomendaciones de CODAES resultó eficaz al lograr la aceptación de un buen porcentaje de los alumnos. Una situación favorable para el equipo de desarrollo fue el interés que despertaron estos materiales entre los alumnos, pues mediante la recomendación entre pares estos materiales se difundieron y fueron explorados y usados por alumnos que al principio no manifestaron interés.

Los resultados también indican que los alumnos incluyeron las unidades de aprendizaje de programación como temáticas factibles para ser explicadas mediante los OA, esta situación abre un espacio de oportunidad para continuar con el desarrollo de OA ya que tendrán buena recepción en el estudiantado.

\section{CONCLUSIONES}

La mayoría de los alumnos concuerdan en que los OA les fueron de gran utilidad para comprender mejor los temas relacionados con estructura de datos como se muestra en la gráfica 5; sin embargo, los resultados del cuestionario 2 no fueron tan favorables como se esperaban. Es posible que la integración
Gráfico 10. Resultado sobre la explicación de los temas

\section{Puntuacion total obtenida en la quinta pregunta las respuestas de los alumnos: ¿Las explicaciones de los temas fueron claros?}

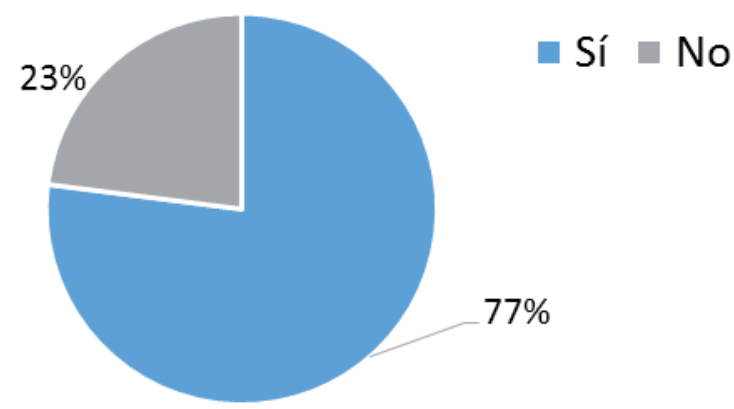

Gráfico 11. Resultado de la pregunta sobre la utilidad de los vídeos

\section{Puntuacion total obtenida en la quinta pregunta las respuestas de los alumnos: ¿Los vídeos le parecieron útiles?}

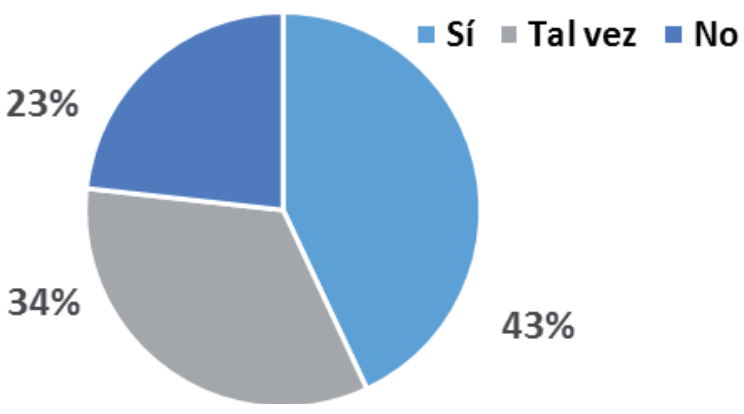

Gráfico 12. Número de alumnos a los cuales les serían útiles $\mathrm{OA}$ relacionados a los temas propuestos

Temas de preferencia para desarrollo de OA segun los Alumnos.

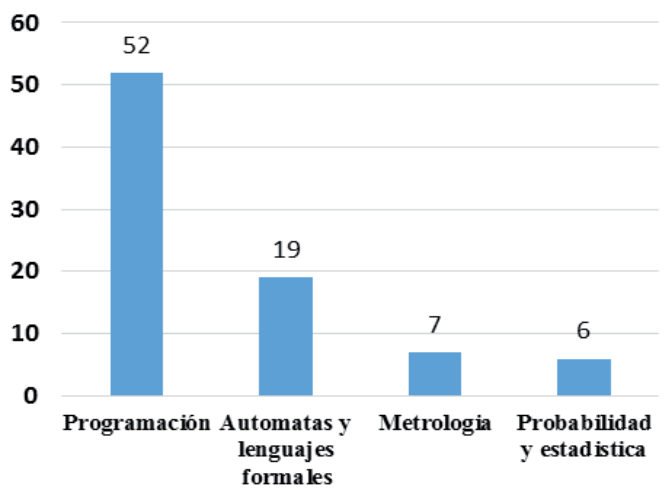


tardía de una cantidad considerable de alumnos haya sido un factor que limitó el buen resultado del último cuestionario. Aun así, es valioso saber que los materiales son valorados por los estudiantes. Los beneficios de tener una evaluación preliminar de los materiales desarrollados son: Facilita la adecuación y mejora de los materiales antes de publicarlos en el curso MOOC.

- Los alumnos conocen con anterioridad los materiales del curso MOOC y al considerarlos de utilidad para su aprendizaje serán una buena referencia para participar en el MOOC.

- El equipo de desarrollo conoce la opinión y percepción de los alumnos usuarios de estos materiales y así visualiza sus oportunidades de mejora en aspectos gráficos, comunicativos y didácticos, esto ayuda a mejorar sus productos de forma consistente.

En este caso se identificó que se pueden desarrollar un mayor número de ejercicios aplicativos con definiciones más amplias puesto que una parte de la población manifestó la necesidad de mayor claridad.

Los resultados sobre la opinión de los alumnos ayudan a identificar las oportunidades de mejora respecto al desarrollo de estos contenidos, los resultados indican que, aunque han sido bien aceptados por los alumnos los objetos de aprendizaje pueden mejorar en los videos, en las explicaciones y en las tareas encomendadas a los alumnos.

Una situación que se conoce a partir de este trabajo es el interés que los alumnos manifiestan sobre tener OA que traten temas relacionados a la programación, pues se sabe por los datos históricos de las calificaciones que el área de la programación les resulta difícil de dominar a los alumnos, es importante saber que ellos ven a los OA como un medio facilitador en su aprendizaje.

Otro aspecto importante es que se ha demostrado que los OA son agradables a los alumnos como una forma interactiva de aprender nuevos temas o comprender mejor los ya estudiados, pero pierden eficiencia al ser recursos limitados en la retroalimentación sobre los temas tratados, pues frecuentemente los alumnos quedan con pequeñas dudas que al final inciden en la resolución de problemas. Esta situación nos permite considerar que la mejor manera de usar estos recursos es con el apoyo de los docentes y además es importante facilitar a los docentes colaborar con el diseño de las actividades y de los cuestionamientos.

\section{REFERENCIAS}

Arias Guerra, Y., y Arias Guerra, Y. (2011). Desarrollo de una biblioteca de estructura de datos avanzadas. Revista Cubana de Ciencias Informaticas, 5, 8. Obtenido de http://www.redalyc. org/articulo.oa?id=378343674003

Astete, M. G. (2014). Videojuegos para apoyar el desarrollo de competencias TIC en. Revista de Educación a Distancia.

Avalos, I., Ramírez, L., Enciso, R., y Flores, M. (2014). Evaluación de la adquisición de competencias: Caso estudiantes del Área de Ciencias Básicas e Ingenierías de la Universidad Autónoma de Nayarit (Vol. vol 12). Revista EducateconCiencia . Recuperado el mayo de 2018, de http://www.tecnocientifica.com. $\mathrm{mx} /$ educateconciencia/index.php/revistaeducate/article/ view $/ 218 / 216$

García-Mundo L, Vargas-Enríquez J. Genero M. y Piattini M (2014) ¿Contribuye el Uso de Juegos Serios a Mejorar el Aprendizaje en el Área de la Informática?, Actas de las XX JENUI. Oviedo, 9-11 de julio 2014, pp 303-310, Universidad de Castilla-La Mancha Ciudad Real, España disponible en: https://upcommons.upc. edu/bitstream/handle/2099/15478/P303ga_cont.pdf

González, F., Moroni, N., y Señas, P. (2004). Visualización de Algoritmos y Programas para el Aprendizaje de Estructuras Arbóreas. VI Workshop de Investigadores en Ciencias de la 
Computación. Disponible en: http://sedici.unlp.edu.ar/handle/10915/21277

Guzmán Valdivia, C. (2013). REPROBATION AND LACK OF INTEREST IN MECHATRONICS ENGINEERING STUDENTS. Venezuela: Revista Orbis. Recuperado el mayo de 2018, de http://www. redalyc.org/html/709/70928419003/

Jiménez Cruz, J. (2017). Integración de un curso MOOC y de un PLN-PLE en un curso presencial sobre fundamentos de la programación. Revista de Educación a Distancia, 53.

López-Escribano, C. (2012). Scratch y Necesidades Educativas Especiales. RED. Revista de Educación a Distancia, 34, 1-14.

OCDE (2017). México - Nota país - Panorama de la educación 2017: Indicadores OCDE. Recuperado el mayo de 2018, de http:// www.oecd.org/education/skills-beyond-school/EAG2017CNMexico-Spanish.pdf

Olabe, X. B. (2015). Pensamiento Computacional a través de la Programación . Revista de Educación a Distancia, 45.

Red CODAES (2014). Documento Base 13 y 14 de octubre del 2014. Colima, Colima. Recuperado el octubre de 2017, de http:// www.codaes.mx/content/micrositios/2/file/Documento_ Base_CODAES.pdf

Red CODAES (2015). Objeto de Aprendizaje. Colima , Colima. Recuperado el noviembre de 2017, de http://www.codaes.mx/ content/micrositios/2/file/GuiaOA-CODAES.pdf

Urbina Nájera, A. (2019). Estrategia tecnológica para mejorar el rendimiento académico universitario. Píxel-Bit. Revista de Medios y Educación, 56, 71-93. http://dx.doi.org/10.12795/ pixelbit.2019.i56.04

Baller, S., Dutta, S. y Lanvin, B. (Edits.) (2016). The Global Information Technology Report 2016. Cornell University. Recuperado el enero de 2018, de http://www3.weforum.org/docs/ GITR2016/WEF_GITR_Full_Report.pdf 